\title{
Retrosplenial Cortex: Possible Role in Habituation of the Orienting Response
}

\author{
Samantha E. Kwon, ${ }^{1}$ Stephen E. Nadeau ${ }^{1,2}$ and Kenneth M. Heilman ${ }^{1,3}$ \\ 'Department of Neurology, Univeristy of Florida College of Medicine, Gainesville, Florida 32601, and '2Geriatric Research, \\ Education, and Clinical Center, and ${ }^{3}$ Neurology Service, Veterans Affairs Medical Center, Gainesville, Florida 32608
}

\begin{abstract}
A patient with a unilateral lesion that included the retrosplenial area had a propensity to attend to contralateral stimuli. To determine whether the retrosplenial-area lesion was inducing this defect, unilateral retrosplenial lesions were produced in rats by surgical aspiration. Animals were assessed preoperatively and postoperatively for orientation and habituation to bilateral simultaneous stimulation in 3 sensory modalities: visual, tactile, and auditory. At each session, the orientation test was terminated upon completion of 5 trials per modality, and the habituation test ended after 4 consecutive response failures or upon completion of 15 trials per modality. Postoperatively, contralateral orientation was not significantly different from ipsilateral orientation, suggesting that there was no neglect. However, there was a significant delay in habituation to contralateral stimulation in postoperative weeks 2 and 3-5. Our results suggest that rats with retrosplenial area lesions have normal orientation but fail to habituate to contralateral stimuli.
\end{abstract}

Valenstein et al. (1987) described a patient (T.K.) who developed severe retrograde and anterograde amnesia following an intracerebral hemorrhage from an arteriovenous malformation located around the splenium of the corpus callosum and extending to the trigone of the left lateral ventricle. After much of the hemorrhage had been resorbed, repeated computed tomographic (CT) and magnetic resonance imaging (MRI) scans revealed a lesion involving the left retrosplenial cortex. Involvement of the underlying cingulum bundle could not be ruled out. T.R. had particularly severe impairment in verbal memory and in recall of new temporal information (Bowers et al., 1988). In addition, he exhibited an unusual orienting behavior to contralateral stimuli (Heilman et al., 1990). He repeatedly complained of a pull to orient to right-sided stimuli, despite a partial right hemianopia. He also demonstrated variable left-sided extinction to bilateral simultaneous visual stimulation and an occasional failure to read the left half of compound words. Even $3 \mathrm{yr}$ after the surgical removal of his arteriovenous malformation and long

\footnotetext{
Received Feb. 5, 1990; revised May 4, 1990; accepted June 18, 1990

This work was supported by the Medical Research Service of the Department of Veterans Affairs. Dr. Kwon was supported as a Medical Student Research Fellow by the American Heart $\Lambda$ ssociation. We wish to thank Donald Walker, Ph.D., for use of his laboratory, Mike King. Ph.D., and Dot Robinson for their kind and expert assistance in histology, Harry Rambo for his technical assistance in behavioral studies, Janet Wootten for her editorial guidance, and Anne Crawford for her preparation of the manuscript.

Correspondence should be addressed to Stephen E. Nadeau, GRECC (182), Veterans Affairs Medical Center, Gainesville, FL 32608-1197.

Copyright (C) 1990 Society for Neuroscience 0270-6474/90/113559-05\$03.00/0
}

after resolution of the visual-field defect, his wife still expressed concern about her husband's propensity to attend to right-sided stimuli.

Disruption of components of Papez's circuit such as the hippocampus has been implicated in amnesia. The retrosplenial area is a part of this circuit. Although retrosplenial damage may be responsible for T.R.'s amnesia, the basis of his unusual attentive behavior remains unclear. T.R. was thoroughly examined with imaging procedures, but in the absence of a post mortem examination, we cannot be certain that there was not damage to structures other than the retrosplenial cortex. To determine whether a retrosplenial-area lesion can induce an attentional asymmetry and to elucidate the nature of T.R.'s unusual behavior, unilateral retrosplenial-area lesions were produced in rats, and the behavioral sequelae were observed.

\section{Materials and Methods}

Subjects. Twelve male Long-Evans hooded rats weighing between 450 and $550 \mathrm{gm}$ were housed on a 12-hr light: dark schedule under standard laboratory conditions and maintained on ad libitum food and water. To minimize freezing and struggling and to maximize responsiveness in the testing position, each rat was handled daily for $3-4$ weeks prior to behavioral testing.

Behavioral testing. The tests used were modified versions of the testing batteries described by Marshall et al. (1980), Crowne and Pathria (1982), and Corwin et al. (1986). Whereas these investigators used a scoring system that summated individual response-strength scores over a number of trials, we counted only the number of orienting responses to bilateral simultaneous stimuli. Each trial was scored as 1, representing an orienting response to a presented stimulus, or 0 , representing a failure to respond. The test animal was gently held from behind by an examiner as it faced a semicircular wall of $35-\mathrm{cm}$ height and $17-\mathrm{cm}$ radius. Only when the head and body were aligned at the midline and directed toward the center of the enclosure was a stimulus presented. Bilateral simultaneous visual, tactile, and auditory stimuli were presented sequentially.

The visual stimulus consisted of 2 shiny, $1-\mathrm{cm}$-diameter silver balls dangling from the ends of a $U$-shaped metal rod. The balls were brought from the rear and presented at eye level at an approximately $6-\mathrm{cm}$ distance and $45^{\circ}$ relative to the midline in each visual field. The balls were dangled until a response was made or until approximately $5 \mathrm{sec}$ had elapsed. A response was defined as a head turn toward the stimulus on either side. This was usually followed by investigatory sniffing. All other responses, such as ignoring the stimulus, turning past the stimulus, or vague head movements, were considered a failure to respond. The tactile stimulus consisted of 2 wooden rods attached posteriorly to form a $U$ shape. The rods were simultaneously brushed agains the vibrissae in a single caudal-to-rostral stroke. They were kept in place just anterior to the vibrissae until a response was made or $2 \mathrm{sec}$ had elapsed. The criteria defining an orienting response were the same as for the visual stimulus. The auditory stimulus was a single click of approximately 114 $\mathrm{dB}$ produced by a single toy clicker held above the body midline from the rear. An orienting response was defined as a quick investigatory head turn of greater than $30^{\circ}$ from midline to either side made within 
$2 \mathrm{sec}$ of the click. Response failures included ignoring the stimulus or movements of less than $30^{\circ}$.

Two separate tests were performed on each examination day. The test for orientation was performed to look for the presence of neglect. This consisted of a total of 15 trials of bilateral simultaneous stimulation comprised of 5 trials each of visual, tactile, and auditory stimuli presented sequentially. To assess habituation, stimuli in a sensory modality were repeatedly presented until the subject either stopped responding or a maximum of 15 trials had been conducted. Habituation was defined as 4 successive response failures. This test was also performed to visual, tactile, and auditory modalities in sequence.

The preoperative-week score represented the sum of scores for 5 successive d. Surgery was performed on the sixth d, and postoperative testing began $48 \mathrm{hr}$ after surgery. The scores for postoperative weeks 1 and 2 also represented score summations over 5 consecutive d. The scores for postoperative weeks 3-5 represented the sum of 5 scores obtained at 4-d intervals over the course of 3 weeks. The animals were tested but not scored during intervening days to keep them accustomed to the testing situation. Pilot studies suggested that most of the operative effect resolved in the late second or early third postoperative weeks.

The behavioral testing and scoring were conducted by 3 investigators unaware of lesion laterality. Two investigators independently scored all postoperative trials. One of these investigators also scored preoperative trials in 5 animals (providing the basis for computation of interrater reliability), and a third investigator scored preoperative trials in all 12 animals. Only those trials in which both examiners agreed as to both the presence and the side of the orienting response were ultimately scored as 1 . All others were scored as 0 . The agreement between both the preoperative and the postoperative scorers was $94 \%$.

Surgery. If an animal tended to respond more to one side preoperatively, the ipsilateral retrosplenial area was lesioned. Anesthesia was achieved with ketamine (Bristol Labs, Syracuse, NY; $90 \mathrm{mg} / \mathrm{kg}$, i.p.) and xylazine (Mobay Corporation, Shawnee, KS; $15 \mathrm{mg} / \mathrm{kg}$, i.p.). The top of the rat's head was shaved, and the animal was placed in a stereotaxic apparatus without ear bars to eliminate any risk of tympanic membranc damagc. Under clcan but not ascptic conditions, a midline incision was made and the skin retracted. Under a surgical microscope, a boneflap was removed with a drill over the anticipated lesion site, exposing the dura mater. After incision of the dura mater, the underlying cortex, extending from approximately $2-9 \mathrm{~mm}$ posterior to the bregma and from the interhemispheric fissure to $2.5 \mathrm{~mm}$ laterally (Paxinos and Watson, 1986), was aspirated with a fine-gauge pipette down to the white matter. The lesion site was gently filled with Gelfoam (Upjohn, Kalamazoo, MI) and the skin closed with wound clips. A single intraperitoneal dose of mannitol (American Regent Labs, Shirley, NY; 1.5 $\mathrm{gm} / \mathrm{kg}$ ) was then administered.

All subjects were killed after the fifth week postsurgery. A lethal dose of pentobarbital was followed by an intracardiac perfusion of $0.9 \%$ saline, then $10 \%$ neutral-buffered formalin. The brains were immediatcly dissectcd out of the cranium and immersed in formalin. The brains were sectioned at $40-\mu \mathrm{m}$ intervals, and every 6 th section was mounted and stained with Cresyl violet. Histologic mapping of each lesion was conducted by an investigator unaware of animal identity and intended lesion laterality.

\section{Results}

Histology. The maximal and minimal extent of the lesions are indicated in Figure 1. There was little variation in lesion size among the 12 rats and no evidence of hippocampal or contralateral hemispheric damage. In 1 animal, the lesion impinged upon the most superficial layer of the superior colliculus. Midgley and Tees (1981) have reported that damage to the deep layers of the superior colliculus is necessary to produce neglect, whereas rats with injury to superficial layers do not have impaired orienting. In 6 animals, the injury encroached anteriorly upon the caudal border of the anterior cingulate (area 24) and the dorsomedial frontal shoulder cortex. Ten animals had evidence of damage to the cingulum bundle, and in 4, the corpus callosum was involved. We were unable to distinguish any behavioral effects of these unintended lesions.

Behavior. The contralateral and ipsilateral orientation and habituation scores for each of the subjects for each preoperative and postoperative week were analyzed. A repeated-measures analysis of variance (ANOVA) of orientation scores was conducted, comparing the effect of operative status, side of operation, and their interaction. There were no significant main effects or interactions, suggesting that unilateral ablation of the retrosplenial area does not produce neglect (Fig. 2).

We performed a repeated-measures ANOVA on the habituation scores to compare opcrative status, side of operation, and their interaction (Fig. 3). There was a main effect for operative status. The unilateral lesion produced a significant overall postoperative delay in habituation for weeks $1(F=23.15, p<$ $0.001), 2(F=17.84, p<0.002)$, and $3-5(F=7.39, p<0.05)$. However, the enhancement of responsiveness attenuated beyond the second postoperative week $(F=18.63, p<0.005)$. There was also a significant interaction between side and operative status for postoperative week $2(F=16.61, p<0.005)$ and weeks 3-5 $(F=11.19, p<0.01)$. Post hoc comparisons for postoperative week 2 revealed a significant delay in habituation to contralateral stimuli relative to ipsilateral stimuli $(t=3.01$, $p<0.02$ ). There was a similar delay of habituation to contralateral stimuli in weeks $3-5(t=2.75, p<0.02)$. A matchedpairs $t$ test comparing contralateral and ipsilateral orientation scores during the preoperative week revealed a propensity to orient to the side ipsilateral to the ensuing lesion $(t=2.20, p=$ 0.05 ). Because the more marked delay in habituation was contralateral, the effect of retrosplenial damage on postoperative contralateral habituation cannot be accounted for on the basis of a preoperative orientation bias.

\section{Discussion}

When a novel stimulus is delivered to an intact organism, the organism will detect the stimulus and direct its attention to the stimulus. Stimulus detection and directed attention are associated with changes in both the somatic (e.g., head and eye turning) and autonomic (e.g., pupil size, heart beat, sweating) nervous system. Stimulus detection, directed attention, and associated behavior comprise the orienting response (Lynn, 1966). Habituation occurs when a meaningless stimulus is repeatedly presented, and the orienting response wanes until the stimulus is no longer attended. While the patient T.R. complained that his attention was being pulled to the side opposite his lesion, he was never systematically studied to learn if this "pull" was related to either a lateralized increase in his orienting response or a failure to habituate. We therefore tested our animals for both orienting and habituation. Our results suggest that, whereas rats with retrosplenial lesions have a normal orienting response to novel stimuli, with repeated stimulation they fail to habituate to stimuli presented contralateral to their lesion. Although a lateralized habituation failure may account for T.R.'s complaint of attentional pulling, his left-sided extinction and occasional failure to read the left side of compound words are difficult to account for on the basis of impaired habituation and indicate some caution in accepting the completeness of the animal model.

To account for the delayed contralateral habituation observed in rats with unilateral retrosplenial lesions, we will consider the nature of habituation, the anatomic structures previously linked with habituation, the anatomic relation of retrosplenial cortex to these structures, and potential mechanisms by which a retrosplenial lesion might affect both habituation and recent memory function as in the patient T.R. 
To explain the neural mechanism underlying habituation, Sokolov (1963) proposed the model-comparator theory, in which the cortex forms a model of repeated stimuli. If the incoming stimulus is novel, there is no model, the reticular activating system is activated, and the animal orients. If the incoming stimulus matches the model, the reticular system is not activated, and habituation takes place. Initially, Sokolov (1963) suggested that the model-comparator process took place in the cortex, but he subsequently suggested that it may take place in the hippocampus (Sokolov, 1975).

Intact orienting to novel stimuli with impaired habituation to repeated nonmeaningful stimuli implies an inability to learn. The hippocampus is well known for its role in memory and learning (Squire, 1987), and a number of studies also suggest that it plays a role in attentional behavior. The hippocampal $\theta$ rhythm occurs in association with cortical desynchronization during a behavioral state of alertness. As alertness subsides, hippocampal $\theta$ is replaced by desynchronized activity, and slow synchronous rhythms appear in the cortex (Green and Arduini, 1954). Hippocampal $\theta$ activity appears in association with the conditioning stimulus in the acquisition phase of a conditioned response, but disappears as the behavior becomes well learned and the conditioning stimulus no longer elicits an orienting response (Grastyan et al., 1959). Kemp and Kaada (1975) also observed that the process of habituation is associated with progressive desynchronization of hippocampal activity. Animals with hippocampal lesions fail to habituate their orienting response to behaviorally irrelevant auditory-tone stimuli (Rogozea and Ungher, 1968) and fail to shift attention to novel stimuli in the presence of non-novel stimuli (Hendrickson et al., 1969). These studies, coupled with data supporting a hippocampal role in memory functions, suggest that the hippocampus may be involved in processes through which a stimulus is determined to be novel (as in the model-comparator theory of Sokolov) and significant (i.e., behaviorally relevant), and that it helps to mediate appropriate orienting responses to novel stimuli and habituation of responses to non-novel, nonsignificant stimuli.

The association of the retrosplenial area with habituation demonstrated by our study and the literature just reviewed linking the hippocampus with processes underlying habituation are paralleled by substantial anatomic data demonstrating extensive connections between the retrosplenial area and the hippocampus. The retrosplenial area is heavily interconnected with the parahippocampal area, subiculum (area 28), presubiculum, and entorhinal area (Meibach and Seigel, 1977; Rosene and Van Hoesen, 1977; Vogt et al., 1979; Vogt and Miller, 1983; Finch et al., 1984; Insanti et al., 1987).

Although our study clearly demonstrates that the retrosplenial area is important for normal habituation, the exact function of the retrosplenial cortex is unknown. The Sokolov model of habituation requires a neural apparatus to provide sensory input regarding the stimulus, a substrate for the internal model of the stimulus, a system that can compare the present stimulus with the model, and a neural apparatus to direct the orienting response. A lesion damaging any one of these structures, or disconnecting them, could plausibly produce either a failure to orient or a failure to habituate.

The hippocampus, by virtue of its involvement in memory function and attentional behavior, may play the role of the comparator between the model and the present stimulus (Vinogradova, 1975). The frontal lobes play a major role in directing and executing the orienting response (Heilman et al., 1987).

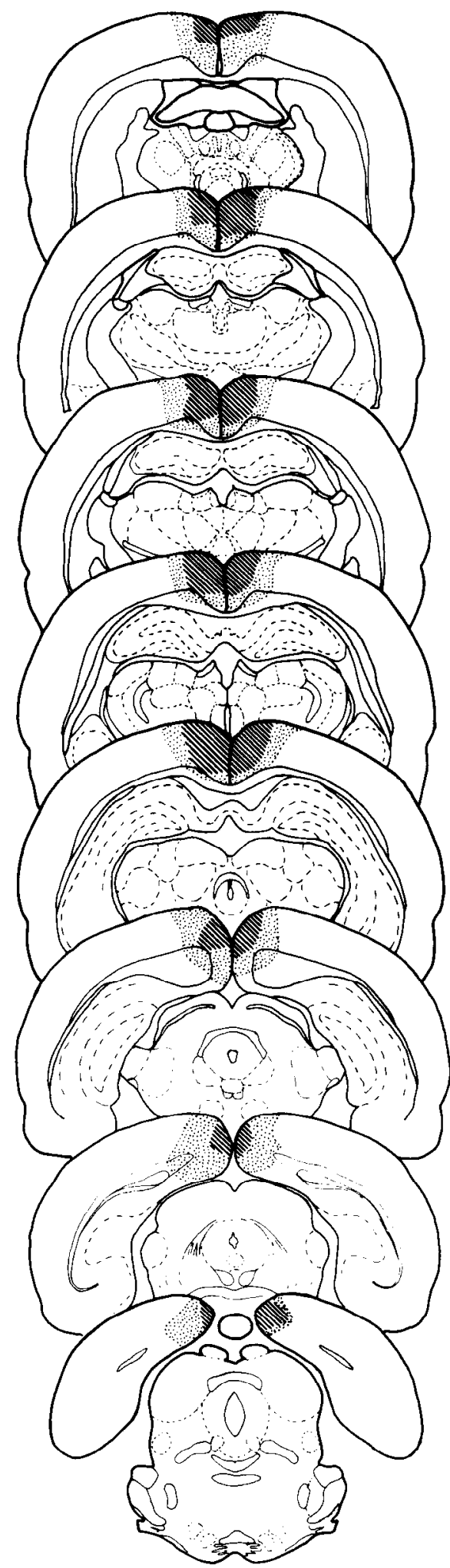

Figure 1. Retrosplenial area lesions. Both right and left unilateral lesions are depicted. The cross-hatching indicates the minimal extent of the lesions, and the stippling, their maximal extent.

There are several possible roles that the retrosplenial cortex could play in this system. A lesion of the retrosplenial area could disconnect the hippocampus from regions of the frontal lobes important for orienting; these areas would then be released, and 


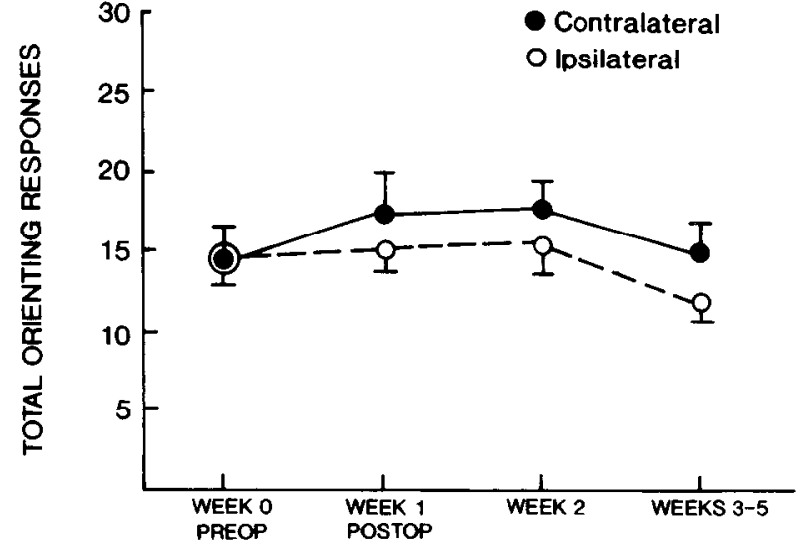

Figure 2. Test for orientation. The contralateral and ipsilateral orienting-response score means of the subjects $(n=12)$ for the orientation trials are represented for each preoperative and postoperative week.

an orienting response would occur indiscriminately, even when the sensory stimulus was clearly no longer novel or significant, that is, a failure of habituation. The retrosplenial cortex of rats has rich reciprocal connections with the anteromedial frontal shoulder cortex, an area that may be homologous to the arcuate (frontal eye field) or the dorsolateral frontal cortex in the monkey (Leonard, 1972; Kolb et al., 1974; Vogt and Miller, 1983; Reep et al., 1984, 1987). Studies in monkeys must be interpreted with caution here because of the limited homology with the rat. Nevertheless, these studies have established interconnections between the retrosplenial area and rostral prefrontal areas 9 and 10 , the orbitofrontal cortex, the prearcuate cortex (areas $8 \mathrm{~A}$ and 45), the dorsal bank of the sulcus principalis (area 46), area 6 , and area 4 (Pandya and Vignolo, 1971; Pandya et al., 1971; Jacobson and Trojanowski, 1977; Vogt et al., 1979; GoldmanRakic et al., 1984). The retrosplenial cortex also has extensive reciprocal connections with the cingulate gyrus, which in turn interconnects with the frontal lobes (Beckstead, 1979; Vogt et al., 1979; Baleydier and Mauguiere, 1980; Pandya et al., 1981; Vogt and Miller, 1983; Mufson and Pandya, 1984).

The concept that a retrosplenial lesion releases the frontal cortex from hippocampal connections finds further indirect support in ${ }^{18} \mathrm{~F}$-deoxyglucose positron-emission tomographic studies of T.R., which showed that the anterior $2 / 3$ of the left hemisphere (ipsilateral to the retrosplenial lesion) was hypermetabolic relative to comparable regions of the right hemisphere (Heilman et al., 1990). There was no clinical or electroencephalographic evidence of seizure activity, and the CT and MRI did not show any right frontal abnormalities. Unfortunately, ${ }^{18} \mathrm{~F}$-deoxyglucose positron-emission tomography does not allow one to distinguish between phasic and tonic activation, and the frontal lobe asymmetries found in T.R. may be related to either an orientation asymmetry or a lateralized failure to habituate.

A second possible mechanism is that the retrosplenial lesion could have disrupted important connections between the corticolimbic orientation/habituation systems and the supcrior colliculus, a structure with which the retrosplenial cortex is also connected (Domesick, 1969) and that also plays a major role in the orienting response (Sparks, 1986).

A third possible effect of a lesion of the retrosplenial cortex on the orienting/habituation system could be the interruption of pathways from association cortices to the hippocampus, thus preventing the hippocampus from comparing the present stim-

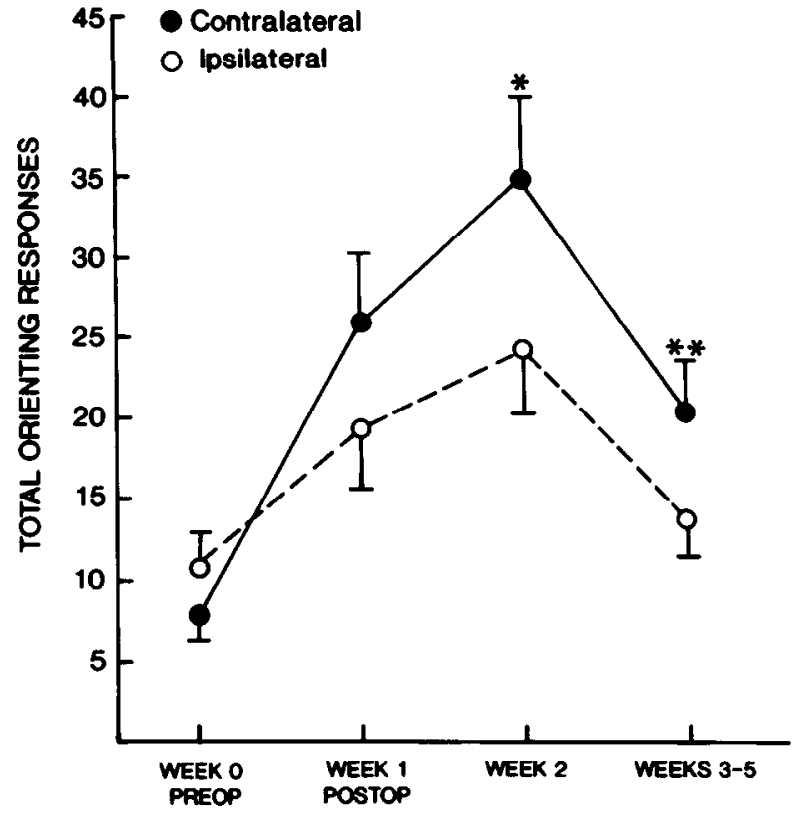

Figure 3. Test for habituation. The contralateral and ipsilateral orienting-response score means of the subjects $(n=12)$ for the habituation trials are represented for each preoperative and postoperative week. Habituation to contralateral stimuli was significantly delayed relative to ipsilateral stimuli as demonstrated by post hoc tests for week 2 (*: $t$ $=3.01, p<0.02)$ and weeks $3-5\left({ }^{* *}: t=2.75, p<0.02\right)$.

ulus with the stored model. This would cause the hippocampus to signal that every stimulus was novel and would in turn lead to repeated orienting responses. Such a lesion could also interrupt hippocampal efferents via the retrosplenial cortex to association cortices, thus preventing both the formation of a model and the encoding of any new information in memory. Either explanation could account for the constellation of features observed in T.R. Primate studies have demonstrated that the retrosplenial area has reciprocal connections with area 7 and the anterior and posterior cingulate (areas 24 and 23; Pandya and Vignolo, 1969; Mesulam et al., 1977; Vogt et al., 1979). In the rat, retrosplenial connections have been demonstrated with the posterior parietal cortex (area 7), primary and associational visual cortex (areas 17 and 18b), temporal auditory cortices (areas 41 and 36), anterior cingulate, infralimbic cortex, and prelimbic cortex (areas 24, 25, and 32; Vogt and Miller, 1983; Reep et al., 1984, 1987; Beckstead, 1979).

Finally, retrosplenial-cortex lesions could affect habituation in still other ways by virtue of the connections of this area with other structures, including the pretectum, the caudate, the putamen, the zona incerta, the midbrain tegmentum, the central gray, claustrum, dorsal and median raphe nuclei, the locus ceruleus, the periventricular nucleus of the hypothalamus, and a number of thalamic nuclei, many of which may be components of attentional systems (Domesick, 1969; Jones and Leavitt, 1974; Mufson and Pandya, 1984; Sripanidkulchai and Wyss, 1986; Thompson and Robertson, 1987).

\section{References}

Baleydier C, Mauguiere F (1980) The duality of the cingulate gyrus in monkey. Neuroanatomical study and functional hypothesis. Brain 103:525-554.

Beckstead RM (1979) An autoradiographic examination of corticocortical and subcortical projections of the mediodorsal projection (prefrontal) cortex in the rat. J Comp Neurol 184:43-62. 
Bowers D, Verfaellie M, Valenstein E, Heilman KM (1988) Impaired acquisition of temporal information in retrosplenial amnesia. Brain Cognition 8:47-66.

Corwin JV, Kanter S, Watson RT, Heilman KM, Valenstein E, Hashimoto A (1986) Apomorphinc has a therapeutic effect on neglect produced by unilateral dorsomedial prefrontal cortex lesions in rats. Exp Neurol 94:683-698.

Crowne DP, Pathria MN (1982) Some attentional effects of unilateral frontal lesions in the rat. Behav Brain Res 6:25-39.

Domesick VB (1969) Projections from the cingulate cortex in the rat. Brain Res 12:296-320.

Finch DM, Derian EL, Babb TL (1984) Afferent fibers to rat cingulate cortex. Exp Neurol 83:468-485.

Goldman-Rakic PR, Selemon LD, Schwartz ML (1984) Dual pathways connecting the dorsolateral prefrontal cortex with the hippocampal formation and parahippocampal cortex in the rhesus monkey. Neuroscience 12:719-743.

Grastyan E, Lissak K, Madarasz I, Donhoffer H (1959) Hippocampal electrical activity during the development of conditioned reflexes. EEG Clin Neurophysiol 11:409-430.

Green HD, Arduini AA (1954) Hippocampal electrical activity in arousal. J Neurophysiol 17:533-557.

Heilman KM, Watson RT, Valenstein E, Goldberg ME (1987) Attention: behavior and neural mechanisms. In: Handbook of physiology, Vol 5 (Mountcastle VB, Plum F, Geiger SR, eds), pp 461-481. Bethesda, MD: American Physiological Society.

Heilman KM, Bowers D, Watson RT, Day A, Valenstein E, Hammond E, Duara R (1990) Frontal hypermetabolism and thalamic hypometabolism in a patient with abnormal orienting and retrosplenial amnesia. Neuropsychologia 28:161-169.

Hendrickson CW, Kimble RJ, Kimble DP (1969) Hippocampal lesions and the orienting response. J Comp Physiol Psychol 67:220227:

Insanti R, Amari DG, Cowan WM (1987) The entorhinal cortex of the monkey: II. Cortical afferents. J Comp Neurol 264:356-395.

Jacobson S, Trojanowski JQ (1977) Prefrontal granular cortex of the rhesus monkey: I. Intrahemispheric cortical afferents. Brain Res 132: 209-233.

Jones EG, Leavitt RY (1974) Retrograde axonal transport and the demonstration of non-specific projections to the cerebral cortex and striatum from thalamic intralaminar nuclei in the rat, cat and monkey. J Comp Neurol 154:349-378.

Kemp IR, Kaada BR (1975) The relation of hippocampal theta activity to arousal, attentive behaviour and somato-motor movements in unrestrained cats. Brain Res 95:323-342.

Kolb B, Nonneman AJ, Singh RK (1974) Double dissociation of spatial impairments and perseveration following selcctivc prefrontal lcsions in rats. J Comp Physiol Psychol 87:772-780.

Leonard CM (1972) The connections of the dorsomedial nuclei. Brain Behav Evol 6:524-541.

Lynn R (1966) Attention, arousal and the orienting reaction. Oxford: Pergamon.

Marshall JF, Berrios N, Sawyer S (1980) Neostriatal dopamine and sensory inattention. J Comp Physiol Psychol 94:833-846.

Meibach RC, Seigel A (1977) Subicular projections to the posterior cingulate cortex in rats. Exp Neurol 57:264-274.

Mesulam M-M, Van Hoesen GW, Pandya DN, Geschwind N (1977) Limbic and sensory connections of the inferior parietal lobule (area
PG) in the rhesus monkey: a study with a new method for horseradish peroxidase histochemistry. Brain Res 136:393-419.

Midgley GC, Tees RC (1981) Orienting behavior by rats with visual cortical and subcortical lesions. Exp Brain Res 41:316-328.

Mufson EJ, Pandya DN (1984) Some observations on the course and composition of the cingulum bundle in the rhesus monkey. J Comp Neurol 235:31-45.

Pandya DN, Vignolo LA (1969) Interhemispheric projections of the parietal lobe in the rhesus monkey. Brain Res 15:49-65.

Pandya DN, Vignolo LA (1971) Intra- and inter-hemispheric projections of the precentral, premotor and arcuate areas in the rhesus monkey. Brain Res 26:217-233.

Pandya DN, Dye P, Butters H (1971) Efferent corticocortical projections of the prefrontal cortex in the rhesus monkey. Brain Res 31: $35-46$

Pandya DN, Van Hoesen GW, Mesulam M-M (1981) Efferent connections of the cingulate gyrus in the rhesus monkey. Exp Brain Res 42:319-330.

Paxinos G, Watson C (1986) The rat brain in stereotaxic coordinates (2d ed). Australia: Academic.

Reep RL, Corwin JV, Hashimoto A, Watson RT (1984) Afferent connections of medial precentral cortex in the rat. Neurosci Lett 44 247-252

Reep RL, Corwin JV, Hashimoto A, Watson RT (1987) Efferent connections of the rostral portions of medial agranular cortex in rats. Brain Res Bull 19:203-221

Rogozea R, Ungher J (1968) Changes in orienting activity of cat induced by chronic hippocampal lesions. Exp Neurol 21:176-186.

Rosene RL, Van Hoesen GW (1977) Hippocampal efferents reach widespread areas of cerebral cortex and amygdala in the rhesus monkey. Science 198:315-317.

Sokolov EN (1963) Perception and the conditioned reflex. Oxford: Pergamon.

Sokolov EN (1975) The neuronal mechanisms of the orienting reflex. In: Neuronal mechanisms of the orienting reflex (Sokolov EN, Vinogradova OS, eds), pp 217-235. Hillsdale, NJ: Erlbaum.

Sparks DL (1986) Translation of sensory signals into commands for control of saccadic eye movements: role of primate superior colliculus. Physiol Rev 66:118-171.

Squire LR (1987) Memory and the brain. New York: Oxford UP.

Sripanidkulchai K, Wyss JM (1986) Thalamic projections to retrosplenial cortex in the rat. J Comp Neurol 254:143-165.

Thompson SM, Robertson RT (1987) Organization of subcortical pathways for sensory projections to the limbic cortex: I. Subcortical projections to the medial limbic cortex in the rat. J Comp Neurol 266:175-188.

Valenstein E, Bowers D, Verfaellie M, Watson R, Day A, Heilman KM (1987) Retrosplenial amnesia. Brain 110:1631-1636.

Vinogradova OS (1975) Functional organization of the limbic system in the process of registration of information: facts and hypotheses. In: The hippocampus: neurophysiology and behavior, Vol 2 (Isaacson RL, Pribram KH, eds), pp 3-69. New York: Plenum.

Vogt BA, Miller MW (1983) Cortical connections between rat cingulate cortex and visual, motor, and postsubicular cortices. J Comp Neurol 216:192-210.

Vogt BA, Rosene DL, Pandya DN (1979) Thalamic and cortical afferents differentiate anterior from posterior cingulate cortex in the monkey. Science 204:205-207. 